\title{
Why the Speed of Light Is Not a Constant
}

\author{
Paul Smeulders \\ Sutton Courtenay, UK \\ Email: paul.smeulders@btinternet.com
}

Received February 12, 2012; revised March 16, 2012; accepted March 25, 2012

\begin{abstract}
A variable Speed of Light is supported by the fact that all direct measurements of that speed are basically flawed, because the "meter per second" is proportional to the Speed of Light. Since it is impossible to measure the Speed of Light directly, any variations of it can only be obtained in an indirect way. It will be shown that the recent Supernovae data are in very good agreement with a universe that is slowly expanding exponentially with a Speed of Light that falls over time, inversely proportionally to the expansion of the universe. It will be shown that the definition of the angular and standard impulse momentum has to be modified to get a consistent expansion of the universe. And that all clocks run inversely proportionally to the red-shift $z+1$. General Relativity remains valid even with a varying Speed of Light and also Quantum Mechanics is unaffected.
\end{abstract}

Keywords: Variable Speed of Light; Expansion of Universe; Conservation of Energy and Angular Impulse Momentum; Supernovae

\section{Introduction}

It is essential that when making a measurement to make sure that the two quantities involved are independent of each other. When the two quantities are shown to be proportional to each other, one always obtains a constant value [1]. It was shown that the " $\mathrm{m} / \mathrm{s}$ " is proportionally to the speed of the electron going around the proton. The latter speed equals the fine-structure constant $\alpha$ times the speed of light. K. Webb et al. [2] have shown that $\alpha$ only changes little over most of the time of the visible universe. Hence measurements of Speed of Light are basically flawed and invalid.

There have been several publications in the past dealing with Variable Speed of Light (VSL) in cosmology [3-6]. Some of the models conserve the mass of the universe and therefore not the energy. And all let the value of the speed of light vary in $\mathrm{m} / \mathrm{s}$. The VSL scheme discussed in this paper will conserve the energy throughout. Angular impulse momentum and impulse momentum will also be conserved, but with some modification in the definition of these quantities in an expanding universe (this has to be done in any case!). And the measured value of the speed of light is constant. So the apparent speed of light is constant!

It will be shown in Section 2, that the Hubble Law fits the Supernovae data in an excellent way when a varying Speed of light is taken into account. This then automatically leads to a slowly expanding universe, with an expansion that is exponentially in time. Such an expansion is structurally very different from a power scaled model [7] leading to a very different universe. Although for small red-shifts the exponential expansion follows a power scaling $a(t)=\left(t / t_{0}\right)^{n}$ with $n=1 / 2$ when $c(t)=c_{0} / a(t)$ and $n$ $=1$ in case $c$ is really constant in time. A power scaling would then lead to a very dense universe $\left(\rho>\rho_{\text {cr }}\right)$ in the case of the VSL and an empty universe for a constant speed of light.

Section 3 will demonstrate that the present definition of the angular impulse momentum leads to orbits that are not proportional to the expansion of the universe and that in order to make a consistent expansion one has to multiply the angular momentum with $z+1$. Then any orbit, including electrons around protons, will scale with $a(t)$.

In Section 4, it is shown that any clock scales inversely proportional to $z+1$. All processes will therefore run faster when going back in time. It is also shown that the Lorentz length scales with $a(t)$ and therefore Relativity remains valid even for a changing Speed of Light.

\section{The Hubble Law}

Over the course of time the Hubble Law has evolved to test models of our universe. Initially Hubble found that the expansion speed was proportional to the measured distance of the objects, leading to the relation of $v=$ H.D. For small red-shifts $z$ the velocity $v=c \cdot z$ with $c$ the speed of light (see N. Wright [7] for more details). If one displays the measured distances of the Supernovae against the product of the redshift $z$ and the velocity of light $c$ one gets a 
curve that can be explained by an expanding empty universe or also by a flat dark energy model [8].

Figure 1 shows the measured distances provided by A. Riess et al. [9] as a function of the red-shift $z$ together with the calculated distance representing an expanding empty universe. Our universe is certainly not empty, but these distances are close to those calculated for a flat darkenergy model [8].

The fact that the speed of light is not necessarily a constant [1] and that a diminishing speed of light also puts the observations further away than a model in which the speed of light is constant, leads to a tempting modification of the Hubble Law by letting $c(t) \cdot z$ being the variable against which to plot the measured distances. The red shift is then a combination of the contribution by the expansion of the universe with scale $a(t)$ and the contribution of the change in the speed of light $c(t)$. It can be readily seen that with a conservation of the Planck constant $\hbar$ the red shift becomes:

$$
z+1=\frac{\lambda_{o b s}}{\lambda_{e m}} \frac{\lambda_{e m}}{\lambda_{0}}=\frac{\lambda_{o b s}}{\lambda_{0}}=\frac{c(t)}{c_{0} a(t)}
$$

The first ratio of the wavelengths is due to the expansion of the universe and the second part related to the change in the speed of light. In case $c(t)=c_{0} / a(t)$ one gets a simple relation between $a(t)$ and the red-shift $z$ : $a(t)=(z+1)^{-1 / 2}$. And of course $c(t)=c_{0} \cdot(z+1)^{1 / 2}$. In this way $c \cdot z=c_{0} \cdot(z+1)^{1 / 2} \cdot z$.

This relation is then given in Figure 2. The result clearly points to the fact that the measured distance has the following relation to the red $\operatorname{shift} z$ :

$$
D_{L}=c_{0} t_{0} \sqrt{z+1}[(z+1)-1]
$$

This should be compared with the calculated one [8]:

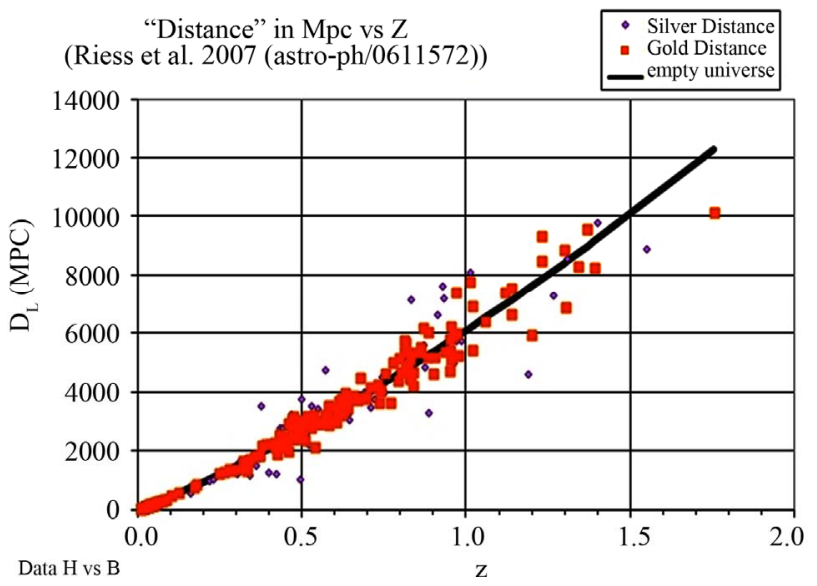

Figure 1. Measured distances of the Supernovae from A. Riess et al. against the measured red shift $z$. Also shown is the calculated distance (solid black curve) against $z$ for an empty expanding universe $a(t)=t / t_{0}$ with $t_{0}=14.4 \mathrm{G}$ year. This calculated distance is very close to the one calculated for a flat dark energy model [8].

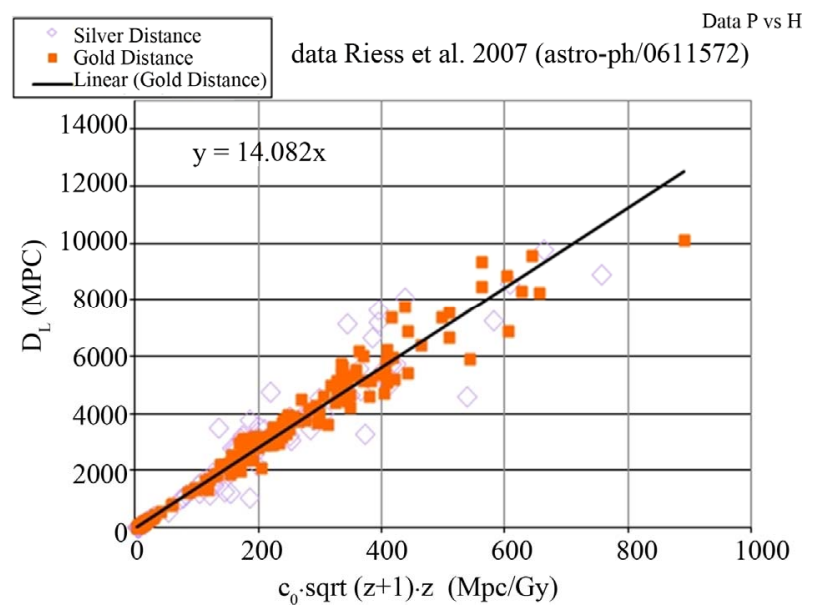

Figure 2. The measured distance $D_{L}$ versus the product $c(t) \cdot z$ in which $c(t)=c_{0} / a(t) . c_{0}$ is the speed of light at present. A remarkable good fit with the data is obtained with a characteristic time $\boldsymbol{t}_{0}$ of $14.082 \mathrm{G}$ year.

$$
D_{\text {now }}=\frac{1}{a(t)} \int_{t}^{t_{0}} \frac{c(t)}{a(t)} \mathrm{d} t=\frac{c_{0} t_{0}}{a(t)} \int_{x}^{1}(z+1) \cdot \mathrm{d} x
$$

with $x=t / t_{0}$.

The Equations (2) and (3) are very similar. This suggests that indeed $a(t)=(z+1)^{-1 / 2}$. From which together with Equation (1) it can be seen that $c(t)=c_{0} \cdot(z+1)^{1 / 2}=$ $c_{0} / a(t)$. Then it follows that $(z+1)$ scales as $e^{(1-x)}$.

\section{Type of Expansion}

Is the exponential expansion the only possible fit?

In principle there are all kind of combinations of $a(t)$ and $c(t)$ possible that match Equations (2) and (3) and satisfy Equation (1).

Equating Equations (2) and (3) and eliminating $c_{0} \cdot t_{0}$, one gets:

$$
\int_{x}^{1}(z+1) \mathrm{d} x=z \cdot(z+1)^{\gamma+\frac{1}{2}}
$$

If one stipulates that $a(t)=(z+1)^{\mathrm{y}}$, one can by varying v get a range of $a(t)$ and $c(t)$ pairs that satisfy Equation (1) and lead to the modified Hubble Law. For instance $\gamma$ $=-1$ represents the expanding universe with a constant speed of light. But all other possibilities lead to a varying speed of light over time. The nature of the red-shift limits the range of gamma to values of: $-1 \leq \mathrm{\gamma} \leq 0$. A universe that would not expand at all is represented by $\mathrm{\gamma}=0$.

Differentiating Equation (4) as function of $x$ yields:

$$
\frac{\mathrm{d} z}{\mathrm{~d} x}=-\frac{(z+1)^{\left(\frac{3}{2}-\gamma\right)}}{\left(\frac{3}{2}+\gamma\right) z+1}
$$

Which gives the following relation between $x$ and $z$ after 
integration:

$$
1-x=\left(\gamma+\frac{3}{2}\right) \frac{(z+1)^{\gamma+\frac{1}{2}}}{\gamma+\frac{1}{2}}-\left(\gamma+\frac{1}{2}\right) \frac{(z+1)^{\gamma-\frac{1}{2}}}{\gamma-\frac{1}{2}}+\frac{1}{\gamma^{2}-\frac{1}{4}}
$$

It should be noted that for $\gamma \approx-1 / 2$ we have $1-x \approx$ $\ln (z+1)$ with a relative error of $(\gamma+1 / 2) \ln (z+1)$.

For $y=0$, this is the case of no expansion, we get:

$$
\sqrt{z+1}=\frac{(5-x)+\sqrt{(5-x)^{2}-12}}{6}
$$

Indeed $x=1$ or $t=t_{0}$ yields $z=0, x=0$ yields $z=0.43$, and $x=-\infty$ leads to $z+1=\infty$.

This latter does agree with the observations that the red-shift always increases when looking back in time.

For $\gamma=-1$, this is the case of $c(t)=c_{0}$, the speed of light is a real constant, we get:

$$
1-x=\frac{4}{3}-\frac{1}{\sqrt{z+1}}\left[1+\frac{1}{3(z+1)}\right]
$$

For $x=1$ one gets again $z=0$, which is fine. This time however one gets $z=\infty$ at $x=-1 / 3$.

Equation (8) has one real root,

$$
z+1=\left[\begin{array}{l}
\sqrt[3]{\left(1+3 x-\sqrt{5+6 x+9 x^{2}}\right) / 2} \\
+\sqrt[3]{\left(1+3 x+\sqrt{5+6 x+9 x^{2}}\right) / 2}
\end{array}\right]^{-2}
$$

This is for small $x+1 / 3$ close to:

$$
z+1 \approx \frac{1}{\left(x+\frac{1}{3}\right)^{2}}
$$

So one has a choice to make:

First of all one should realize that the modified Hubble Law required that $c(t)=c_{0} / a(t)$ and that therefore already we have strong evidence for $\gamma=-1 / 2$.

Secondly, looking again at Equation (2) that was found from the measurements:

$$
D_{L}=c_{0} t_{0} \sqrt{z+1}[(z+1)-1]
$$

And the definition of a measured distance (3):

$$
D_{\text {now }}=\frac{c_{0} t_{0}}{a(t)} \int_{x}^{1}(z+1) \mathrm{d} x
$$

The most obvious connection to make is to put:

$$
\sqrt{z+1}=1 / a(t) \text { and } \int_{x}^{1}(z+1) \mathrm{d} x=(z+1)-1
$$

This then leads directly to: $c(t)=c_{0} / a(t)$ and $z+1=e^{(1-x)}$

It is the most straightforward relation that we can extract from Equations (2) and (3), but in principle it does not exclude other options discussed above.

The "obvious" connection leads to an expansion growing exponentially in time: its scale factor is $a(t)=\exp \left[\left(t / t_{0}-1\right) / 2\right]$. The growth is slow with an e-folding time of $28.2 \mathrm{G}$ year.

Thirdly as shown in the discussion in Section 5, there is supporting evidence that the sizes of the galaxies scale as $(z+1)^{m}$, with $-1<m<0$. So that $\gamma=-1 / 2$.

It is interesting to note that for small $z,\left(t\right.$ close to $\left.t_{0}\right)$ one can expands $a(t)$ and obtains a power scaling of $a(t)$ $\approx\left(t / t_{0}\right)^{1 / 2}$. This would be the power scaling for a dense universe with $\rho>\rho_{\text {cr }}$ the latter being the critical density at which the expansion of the universe is just not stopping [8]. So by letting the speed of light relax over time the universe can have density compared to the power scaling of $a(t)=\left(t / t_{0}\right)$ which fitted the data so good for the constant speed of light model, but had zero density power scaling.

An exponential growth is structurally different from a power scaling. A power scaling has a beginning at $t=0$, an exponential growth has no beginning $(t=-\infty$ is the start of all) and it has no end. $t_{0}$ is just an e-folding time for $(z+1)$ and $2 * t_{0}$ for $a(t)$. But for sure the beginning and the end of the universe are both well outside the measurements. It may well be that the beginning was linear and the end will be in a saturation state, in this case the exponential expansion can no longer be applied.

The quality of the fit of the exponential growth model to the Supernovae data is remarkable good. This is shown in Figure 3.

There is no systematic difference between the measured distances and the calculated one. In the case for the zero density power scaling [9] there is a positive difference. An accelerating dark-energy model was required to explain this difference.

An expansion accelerating in recent times is of course in agreement with an exponential growing expansion. But the latter came "naturally" out of the measured data itself.

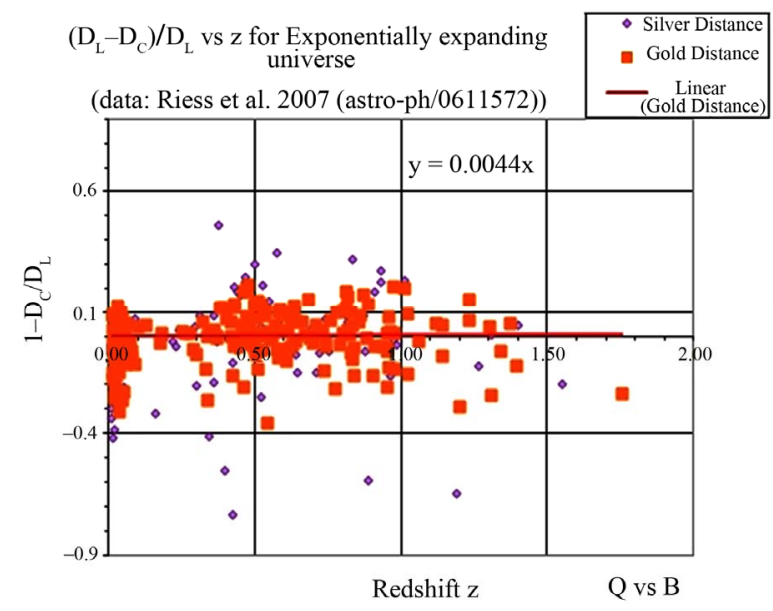

Figure 3. The relative difference between the measured and calculated distances. The red line is the straight line fit to the Gold data of A. Riess et al. Note: $z=1.755$ is $14.5 \mathrm{G}$ years ago. 
It is clear, that an exponentially growing expansion needs an "explanation" too in the form of dark energy that drives the growth.

\section{The Expansion and Conservation Laws}

It is necessary to check whether conservation laws remain valid in these changing conditions. It should be noted that this section is valid for all combinations of $a(t)$ and $c(t)$, i.e. whether the speed of light is a real constant or not.

The first law to check is the energy conservation law for e.g. a planet of mass m orbiting a star with mass $M$ at a distance $r$. The condition that the planet is in an orbit around the star gives the following condition:

$$
v^{2}=-G \frac{M}{r}
$$

The energy is the kinetic plus potential energy, which equals:

$$
\begin{aligned}
E_{k i n}+E_{p o t}-E_{p o t}^{\infty} & =\frac{1}{2} m v^{2}-G \frac{m M}{r} \\
& =\frac{3}{2} m v^{2}=\frac{3}{2} m c^{2} \cdot\left(\frac{v}{c}\right)^{2}
\end{aligned}
$$

With changing $a(t)$ and $c(t)$ energy is thus conserved if the ratio of $v / c$ is conserved, since from relativity $m c^{2}$ is conserved. This implies too that the relativistic energies are conserved.

The law of angular impulse momentum is:

$$
P=m \cdot v \cdot r=\frac{m v^{2}}{\omega}
$$

Since $m v^{2}$ is conserved, angular impulse momentum conservation would mean conservation of $\omega$. Also it would follow then that $r / c$ is conserved and this implies:

$$
r(t)=c(t) \frac{r_{0}}{c_{0}}=(z+1) \cdot a(t) \cdot r_{0}=a^{1+1 / \gamma}(t) \cdot r
$$

It is clear that for $-1<\gamma<0$ Equation (14) leads to serious problems with the expansion condition that $r(t)$ should be equal to $r_{0} \cdot a(t)$.

Let us see how one can redefine the angular impulse momentum in such a way that the latter is conserved and the orbit can expand in the correct way. Put:

$$
P=m v r \cdot f(t)=m c^{2} \frac{v}{c} a(t) r_{0} \frac{f(t)}{c}=m c^{2} \frac{v}{c} r_{0} \frac{1}{c_{0}}
$$

This gives:

$$
f(t)=\frac{c(t)}{c_{0} a(t)}=z+1
$$

One could state that: $\boldsymbol{P}=m c^{2} \cdot\left[\frac{\boldsymbol{v}}{c} \times \frac{\boldsymbol{r}}{a}\right]$ is conserved and define that is the angular momentum (which it is in our time). This leads to the following orbital frequency:

$$
\omega(t)=\omega_{0} \frac{c(t)}{c_{0} a(t)}=\omega_{0}(z+1)
$$

The same logic applies for the hydrogen atom. The angular impulse momentum is:

$$
m_{e} v \cdot a_{0}(t) \cdot \frac{c(t)}{c_{0} a(t)}=h
$$

It then follows that the Bohr radius is:

$$
a_{0}(t)=h \frac{c_{0}}{m_{e} c^{2} \alpha} a(t)=a_{0}\left(t_{0}\right) \cdot a(t)
$$

With this definition of the angular impulse momentum, conservation implies that the scaling with the expansion scale factor $a(t)$ is maintained for both planet orbits and atoms.

The conservation of the electric charge has to be adapted to the expansion too.

The force balance in the hydrogen atom stipulates that:

$$
\frac{e^{2}}{\varepsilon_{0} a_{0}}=m c^{2} \alpha^{2}
$$

Therefore:

$$
\frac{e^{2}}{\varepsilon_{0}}(t)=m c^{2} \alpha^{2} a_{0} \cdot a(t)=\frac{e^{2}}{\varepsilon_{0}}\left(t_{0}\right) \cdot a(t)
$$

And since $\varepsilon_{0} \mu_{0}=1 / c^{2}$ one may assume that $\varepsilon_{0}(t)=$ $\varepsilon_{0}\left(t_{0}\right) \cdot c_{0} / c(t)$. This leads to:

$$
e^{2}(t)=e^{2}\left(t_{0}\right) \cdot \frac{c_{0} a(t)}{c(t)}=e^{2}\left(t_{0}\right) \cdot \frac{1}{z+1}
$$

\section{The Clocks and Expansion}

Let us take as atomic clock the time it takes for the electron to go around the proton:

$$
\tau_{0}(t)=2 \pi \frac{a_{0}(t)}{v(t)}=\tau_{0}\left(t_{0}\right) a(t) \frac{c_{0}}{c(t)}=\frac{\tau_{0}\left(t_{0}\right)}{z+1}
$$

Also the orbit time for a planet around a star scales like that: Equation (17).

How does the pendulum scales? One has to know how the gravitational constant scales with $c$ and $a(t)$. Energy conservation gives the following relation:

$$
G(t)=G_{0} \frac{m_{0}}{m} \frac{M_{0}}{M} \frac{r}{r_{0}}=G_{0}\left(\frac{c(t)}{c_{0}}\right)^{3} \cdot \frac{c(t) a(t)}{c_{0}}
$$

In our case of $c(t) \cdot a(t)=c_{0}$ we get that $G$ scales with the third power in $c$. But also in case of a constant speed of light one has to take into account that $G$ still scales then with $a(t)$.

The pendulum can now be calculated: 


$$
\tau_{p}(t)=2 \pi \sqrt{\frac{\ell}{g}}=2 \pi \sqrt{\frac{\ell_{0} a(t) c_{0}^{3} r_{0}^{2}}{G_{0} c^{3}(t) M_{0}}}=\frac{\tau_{p}\left(t_{0}\right)}{z+1}
$$

We can conclude that the clock of the pendulum, the orbit period of planets and the orbit period of the electron all change at the same rate in time: inversely proportional to the red-shift $z+1$. Note that also for a really constant speed of light the clocks would still scale like that too.

This dependence of the clocks has implications for the Lorentz equation and so on Relativity. It can be seen that Relativity is unaffected by the expansion of the universe.

The Lorentz equation is:

$$
x^{2}+y^{2}+z^{2}-c^{2} \tau^{2}=a^{2}(t)\left(x_{0}^{2}+y_{0}^{2}+z_{0}^{2}\right)-\frac{c^{2} c_{0}^{2} a^{2}(t)}{c^{2}} \tau_{0}^{2}
$$

This just states that the Lorentz length too is multiplied by $a(t)$. Since also all ratios of $(v / c)$ are conserved, it follows that Relativity remains unaltered.

Also Quantum mechanics are unaffected. It can readily be seen that all energy levels of the Hydrogen atom are in units of $m c^{2} \alpha^{2}$, which remain unchanged.

\section{Discussion}

There exists tempting supporting data for the exponential scaling of the expansion. It has been shown that the galaxy size (in kpc) is a function of the red-shift $z$ [10]. The data in Figure 5 of reference [10] show that the size of galaxies scales as $(1+z)^{m}$, with $m$ between 0 and -1 . For $m=-0.5$ the galaxies would scale as the scale factor of the expanding universe, namely as $a(z)=(1+z)^{-0.5}$. This then supports the exponential expansion.

One can then conclude that starting from 50 Gyears ago $(z=5)$ the universe has been expanding exponentially.

\section{Conclusion}

The exponential scaling law gives a very good match to the Supernovae observations. Exponential expansion requires a driving force. Dark Energy must be there. Power scaling laws are not very suitable to describe the evolution of the universe. Exponential expansion puts the Big Bang far back in time, but this is outside the measurements where we do not really know whether the exponential expansion remains valid. A modification of the definition of the angular impulse momentum is required in order to get a consistent expansion of the universe even in case of a constant speed of light. The clocks in the universe scale inversely proportional to the red-shift $z$ +1 .

\section{Acknowledgements}

The author would like to thank the Mediterranean Institute of Fundamental Physics i.e. Prof. A. Kavokin for his great interest in and support for this controversial topic. Also the author is much in debt to Dr. A. Riess for making the Supernovae data available in his publication.

\section{REFERENCES}

[1] P. Smeulders, "The Measure of the Speed of Light," Superlattices and Microstructures, Vol. 43, No. 5-6, 2008, pp. 651-654. doi:10.1016/j.spmi.2007.07.007

[2] J. K. Webb, et al., "Further Evidence for Cosmological Evolution of the Fine Structure Constant," Physical Review Letters, Vol. 87, No. 9, 2001, Article ID 091301. doi:10.1103/PhysRevLett.87.091301

[3] A. Albrecht and J. Magueijo, "Time Varying Speed of Light as a Solution to Cosmological Puzzles," Physical Review D, Vol. 59, No. 4, 1999, Article ID 043516. doi:10.1103/PhysRevD.59.043516

[4] J. D. Barrow, "Varying Constants," Philosophical Transactions of the Royal Society London, Vol. A363, 2005, pp. 2139-2153.

[5] J. C. Gimenez, "A Simple Cosmological Model with Decreasing Light Speed," 2003, arXiv:astro-ph/0310178.

[6] J. W. Moffat, "Superluminary Universe: A Possible Solution to the Initial Value Problem in Cosmology," International Journal of Physics D, Vol. 2, No. 3, 1993, pp. 351365. doi:10.1142/S0218271893000246

[7] E. Wright http://www.astro.ucla.edu/ wright/cosmolog.html

[8] E. Wright http://www.astro.ucla.edu/ wright/sne_cosmology.html

[9] A. Riess, et al., "New Hubble Space Telescope Discoveries of Type la Supernovae at $z \geq 1$ : Narrowing Constraints on the Early Behavior of Dark Energy," Astrophysical Journal, Vol. 659, No. 1, 2007, pp. 98-121. doi: $10.1086 / 510378$

[10] J. Bouwens, et al., "Galaxy Size Evolution at High Redshift and Surface Brightness Selection Effects: Constraints from the Hubble Ultra Deep Field," Astrophysical Journal, Vol. 611, No. 1, 2004, pp. L1-L4. doi:10.1086/423786 